\title{
CONTRACTIBLE POLYHEDRA IN PRODUCTS OF TREES AND ABSOLUTE RETRACTS IN PRODUCTS OF DENDRITES
}

\author{
SERGEY A. MELIKHOV AND JUSTYNA ZAJA̧C \\ (Communicated by Alexander N. Dranishnikov)
}

\begin{abstract}
We show that a compact $n$-polyhedron PL embeds in a product of $n$ trees if and only if it collapses onto an $(n-1)$-polyhedron. If the $n$ polyhedron is contractible and $n \neq 3$ (or $n=3$ and the Andrews-Curtis Conjecture holds), the product of trees may be assumed to collapse onto the image of the embedding.

In contrast, there exists a 2-dimensional compact absolute retract $X$ such that $X \times I^{k}$ does not embed in any product of $2+k$ dendrites for each $k$.
\end{abstract}

\section{INTRODUCTION}

All spaces shall be assumed to be metrizable. By a compactum we mean a compact metrizable space. A finite-dimensional compactum is an $A N R$ if and only if it is locally contractible, and an absolute retract $(A R)$ if and only if it is a contractible ANR (see [5]). A one-dimensional compact AR is called a dendrite, and a one-dimensional compact ANR is called a local dendrite. An arbitrary connected one-dimensional compactum is sometimes called a curve.

Theorem 1.1 (Nagata-Bowers [39, [7; see also [46], 47], 2]). Every n-dimensional compactum $X$ embeds in $D^{n} \times I$, where $D$ is a certain dendrite.

It is well-known that every dendrite embeds in the 2-cube $I^{2}$; thus Theorem 1.1 may be viewed as an improvement of the classical Menger-Nöbeling-Pontriagin theorem that every $n$-dimensional compactum embeds in the $(2 n+1)$-cube $I^{2 n+1}$. Theorem 1.1 is trivial in the case where $X$ is a polyhedron:

Theorem 1.2. Every compact $n$-polyhedron embeds in a product of $n$ trees and $I$.

Proof. Given a triangulation $K$ of the given polyhedron $X$, let $S_{i}$ be the set of all vertices of the barycentric subdivision $K^{\prime}$ that are barycenters of $i$-simplices of $K$. The simplicial map $K^{\prime} \rightarrow S_{0} * \cdots * S_{n}$ is clearly an embedding. Hence $X$ embeds in $I * S$, where $S=S_{1} * \cdots * S_{n}$. Next, $I * S=p t * C S$ is homeomorphic to $p t *(C S \cup S \times I)=I \times C S$. Finally, the cone $C S$ is homeomorphic to the product of $n$ trees $C S_{1} \times \cdots \times C S_{n}$.

Received by the editors May 17, 2011 and, in revised form, September 29, 2011; October 7, 2011; and October 8, 2011.

2010 Mathematics Subject Classification. Primary 54C25, 57Q35; Secondary 55P57, 06A07, 57M20, 55M15.

The first author is supported by Russian Foundation for Basic Research Grant No. 11-0100822, Russian Government project 11.G34.31.0053 and Federal Program "Scientific and ScientificPedagogical Staff of Innovative Russia" 2009-2013. 
The above argument yields an explicit embedding of every compact $n$-polyhedron in $I^{2 n+1}$, which we have not seen in the literature. This is strange, for a part of this construction is certainly well-known; it yields

Proposition 1.3 (17, 24]). The cone over every compact n-polyhedron embeds in a product of $n+1$ trees.

Theorem 1.4 (Borsuk-Patkowska [6]). The $n$-sphere $S^{n}$ does not embed in any product of $n$ dendrites, for each $n \geq 0$.

Another proof of Theorem 1.4 is given by the easy part of our Corollary 1.9 below.

Theorem 1.5 (Gillman-Matveev-Rolfsen [17]). Every compact connected PL $n$ manifold with nonempty boundary embeds in a product of $n$ trees.

This was originally a consequence of Proposition 1.3 along with a "reconstruction theorem" announced in [17. Another proof of Theorem 1.5 is given by our Corollary 1.9 below, albeit the trees that it produces need not be cones over finite sets.

Nagata's original motivation for considering embedding into products of 1dimensional spaces was related to dimension theory (see [39]). Borsuk's proof of the 2-dimensional case of Theorem 1.4 was a solution to Nagata's problem; on the other hand, the author learned from W. Kuperberg, a student of Borsuk who has generalized Theorem 1.4 29, that Borsuk saw this result as a part of his work on the problem of uniqueness of decomposition of ANRs into products. Yet another motivation for embedding into products of trees was the Poincaré Conjecture (now also known as Perelman's Theorem):

Theorem 1.6. (a) (Gillman [16]) If a compact acyclic 3-manifold embeds in the product of a tree and $I^{2}$, then it is collapsible.

(b) (Zhongmou [54]) Every compact connected 3-manifold with nonempty boundary embeds in the product of two triods and $I$.

A discussion of further results in the theory of embeddings into products of dendrites (or curves) can be found in the recent paper [24], which itself is a significant addition to this theory (see also additional details in [25]). We should mention

Theorem 1.7 (Koyama-Krasinkiewicz-Spież 24]). There exists a 2-polyhedron that collapses onto a product of two graphs but does not embed in any product of two graphs. Yet it embeds in a product of two curves.

The 2-polyhedron in question is $\Theta \times \Theta \underset{J=I \times\{0\}}{\cup} I \times I$, where $\Theta$ is the suspension over the three-point set, and the arc $J$ lies in the interior of a 2-cell of $\Theta \times \Theta$ apart from one endpoint, which lies in a "corner" of that 2-cell.

\section{A. Embedding contractible polyhedra in products of trees.}

Theorem 1.8. Every collapsible compact n-polyhedron PL embeds in a product of $n$ trees. Moreover, the product of trees collapses onto the image of the embedding.

The embeddability in the 2-dimensional case is due to Koyama, Krasinkiewicz and Spież 24. The principal additional ingredient in our proof of the general 
case is the Fisk-Izmestiev-Witte lemma [15, Lemma 57], [22, [52] (see also [19, Lemma 3.1], 10]), which asserts that for every finite set $C$ (the 'palette') of cardinality $\# C \geq d+1$, every $C$-colored combinatorial $(d-1)$-sphere is color-preserving isomorphic to the boundary of a $C$-colored combinatorial ball. (A simplicial complex is $C$-colored if its vertices are colored by the elements of $C$ so that no edge connects two vertices of the same color.)

In particular, this lemma implies that if a triangulation of $S^{2}$ admits a 4-coloring, then it extends to a triangulation of the 3-ball where the link of every interior edge is (combinatorially) an even-sided polygon. As observed by R. D. Edwards and others in the 1970s, the converse to this also holds: every such triangulation of the 3 -ball has a 4-colorable boundary (see references in [22]).

The 2-dimensional case of Theorem 1.8 involves only the trivial case $d \leq 1$ of the Fisk-Izmestiev-Witte lemma.

Corollary 1.9. Let $P$ be a compact n-polyhedron. The following are equivalent:

(i) P PL embeds in a product of $n$ trees;

(ii) P PL embeds in a product of an $(n-1)$-polyhedron and a tree;

(iii) $P$ collapses onto an $(n-1)$-polyhedron;

(iv) P PL embeds in a collapsible compact n-polyhedron.

Here (iv) $\Rightarrow$ (i) follows from Theorem 1.8 , (i) $\Rightarrow$ (ii) is obvious, (ii) $\Rightarrow$ (iii) is easy (see below), and to see that (iii) $\Rightarrow$ (iv) it suffices to note that if $P$ collapses onto $Q$, then the amalgamated union $P \cup_{Q} C Q$ is collapsible, where $C Q$ is the cone over $Q$.

Alternatively, (i) $\Rightarrow($ iv) is obvious, and another proof of (iii) $\Rightarrow(\mathrm{i})$ is given in $\S 2$.

Proof of $($ ii $) \Rightarrow($ iii $)$. Suppose that $P$ is embedded in $R \times T$, where $R$ is an $(n-1)$ polyhedron and $T$ is a tree, and $P$ does not collapse onto any $(n-1)$-polyhedron. Let $P_{0}$ be a triangulation of $P$. Then $P_{0}$ collapses onto a (generally nonunique) simplicial complex $Q_{0}$ that does not collapse onto any proper subcomplex. Then $Q_{0}$ has no free faces, and it follows that $Q:=\left|Q_{0}\right|$ does not collapse onto any proper subpolyhedron. By the hypothesis, $Q$ is of dimension precisely $n$. The projection $f: Q \subset R \times T \rightarrow R$ can be triangulated by a simplicial map $Q_{1} \rightarrow R_{1}$. Let $p$ be a point in the interior $U$ of a top-dimensional simplex of $R_{1}$ such that the corresponding fiber $F:=f^{-1}(p)$ is of dimension precisely 1 . The projection $F \subset R \times T \rightarrow T$ is an embedding, so $F$ is a forest. Thus $F$ collapses onto a finite set but is not a finite set itself, so it must have a free vertex. On the other hand, $f^{-1}(U) \cong F \times U$ by Pontryagin's lemma [40, Proposition C], [51, Theorem 1.3.1] (see also [11, §5]). Hence $Q_{1}$ has a free face. Thus $Q$ collapses onto a proper subpolyhedron, which is a contradiction.

Corollary 1.10 (Koyama-Krasinkiewicz-Spież [24]). An acyclic compact 2-polyhedron $P$ embeds in a product of two trees if and only if $P$ is collapsible.

Remark 1.11. Let $P$ be a compact polyhedron with $H^{1}(P)=0$. If $P$ embeds in a product of $n$ graphs then it embeds in a product of $n$ trees, namely in the product of (appropriate compact subtrees of) the universal covers of the $n$ graphs. Thus "trees" can be replaced with "graphs" in Corollary 1.10 in accordance with 24]. (In fact, it was shown in 24] that an acyclic noncollapsible compact 2-polyhedron does not embed in any product of two curves.) 
Corollary 1.12. Let $P$ be an $n$-polyhedron. For $n \neq 3$, the following are equivalent:

(i) some product of $n$ trees collapses onto a PL copy of $P$;

(ii) $P$ collapses onto a contractible $(n-1)$-polyhedron;

(iii) some collapsible compact n-polyhedron collapses onto a PL copy of $P$.

For $n=3$, the same holds with "contractible" replaced by "3-deformable to a point".

A polyhedron $P$ is said to be $n$-deformable to a polyhedron $Q$ if they are related by a sequence of collapses and expansions (i.e. the inverses of collapses) through polyhedra of dimensions $\leq n$. The Andrews-Curtis Conjecture asserts that all contractible 2-polyhedra 3-deform to a point (see 1, 32]). Among its motivations (cf. Curtis [13, §2]) we mention that it would imply 1 that every contractible 2polyhedron PL embeds in $I^{4}$.

Proof. (iii) $\Rightarrow$ (i) follows from Theorem 1.8 and (i) $\Rightarrow$ (ii) follows from Corollary 1.9. To prove (ii) $\Rightarrow$ (iii), suppose that $P$ collapses onto an $(n-1)$-polyhedron $Q$, and either $Q$ is contractible, or $n=3$ and $Q$ 3-deforms to a point. Then by a result of Kreher-Metzler and Wall, there exists an $(n-1)$-polyhedron $R$ such that $R$ collapses onto a PL copy of $Q$ and $R \times I$ is collapsible [28, Satz 1a, Satz 1] (see also [1, §XI.4] for an outline of Kreher and Metzler's proof in English). Let $S$ be the amalgamated union $P \cup_{Q=Q \times\{0\}} R \times I$. Then $S \searrow R \times I \searrow p t$ and $S \searrow P \cup_{Q} R \searrow P$.

Remark 1.13. For each $n \geq 3$ it is easy to construct a noncollapsible $n$-polyhedron that collapses onto a contractible $(n-1)$-polyhedron (e.g. $I^{n} \vee$ cone $(f)$ will do, where $f$ is any degree $0 \mathrm{PL}$ surjection $S^{n-2} \rightarrow S^{n-2}$ ). A more interesting example is due to M. M. Cohen, who constructed for each $n \geq 4$ a contractible $(n-1)$-polyhedron $Q$ such that $Q \times I$ is not collapsible [12]. Other constructions (with very different proofs) are now known: $P \times I^{k-2}$ is not collapsible if $P$ is the suspension over a $(k-1)$-dimensional spine of a non-simply-connected homology $k$-sphere [3], and $P \times I^{q}$ is not collapsible if $P$ is a certain " $(3 q+6)$-dimensional dunce hat" [8].

A free deformation retraction of a space $X$ onto a subspace $Y$ is a homotopy $h_{t}: X \rightarrow X$ starting with $h_{0}=\mathrm{id}$, ending with a retraction $h_{1}$ of $X$ onto $Y$, and such that $h_{t} h_{s}=h_{\max (s, t)}$ for all $s, t \in[0,1]$. A space is freely contractible if it freely deformation retracts onto a point. Collapsibility is known to be strictly stronger than topological collapsibility [3], 8] and consequently strictly stronger than free contractibility; however, in the case of 2-polyhedra the three notions are equivalent [21].

Conjecture 1.14. A compact $n$-polyhedron collapses onto an $(n-1)$-polyhedron if and only if it freely deformation retracts onto an $(n-1)$-polyhedron.

Remark 1.15. The proof of Theorem 1.8 involves a (not-straightforward) construction of a collapsible cubulation of the given collapsible polyhedron, which might be of interest in its own right. Another such construction (a more straightforward one) has been used to characterize collapsible polyhedra in the language of abstract convexity theory [48] and to establish the 'only if' part of Isbell's conjecture: a compact polyhedron is collapsible if and only if it is injectively metrizable [30, [49, Chapter VI]. (Isbell himself proved that the two conditions are equivalent for 2-polyhedra [21.)

\footnotetext{
${ }^{1}$ By general position every 2-polyhedron $P$ immerses in $I^{4}$ and therefore embeds in a 4-manifold $M$. Let $N$ be a regular neighborhood of $P$ in $M$. If $P$ 3-deforms to a point, then the double of $N$ is the 4-sphere (see [1, Assertion (59) in Ch. I]).
} 
1.B. Embedding absolute retracts in products of dendrites. A map $f: X \rightarrow$ $Y$ is called an $\varepsilon$-map with respect to some metric on $X$ if every point-inverse $f^{-1}(p t)$ is of diameter at most $\varepsilon$. A compactum $X$ is said to quasi-embed in a space $Y$ if for some (or equivalently, every) metric on $X$, it admits an $\varepsilon$-map into $Y$ for each $\varepsilon>0$. We refer to [42] for a definitive discussion of the (quite subtle) difference between embeddability and quasi-embeddability of compact polyhedra in $I^{m}$.

Our paper was originally motivated by the following problem.

Problem 1.16 (Koyama, Krasinkiewicz, Spież [25]). Suppose that $X$ is a compactum, quasi-embeddable in the $n$th power of the Menger curve. Can $X$ be embedded there?

This problem appears as Problem 1.4 in $[25$ with the following comments: "Our next problem is of great interest, we believe it has affirmative solution."

In the present paper, we shall prove

Theorem 1.17. There exists a 2-dimensional compact $A R X$ such that $X \times I^{k}$ quasi-embeds in a product of $2+k$ dendrites but does not embed in any product of $2+k$ curves, for each $k \geq 0$.

The proof of the higher-dimensional (i.e. $k \geq 1$ ) case is similar to (and only three lines longer than) the proof of the two-dimensional case. Similar arguments show that the Cartesian power $X^{k}$ quasi-embeds in a product of $2 k$ dendrites but does not embed in any product of $2 k$ curves.

Remark 1.18. A few months after we shared our proof of the two-dimensional case of Theorem 1.17 with J. Krasinkiewicz and S. Spiez, they found their own solution of Problem [1.16 [27]. Compared to ours, it is amazingly simple (modulo their previous work with A. Koyama) - at least when slightly modified as follows.

The dunce hat $D$ [53] (also known as the Borsuk tube [4], 27]) is easily seen to be the quotient of a collapsible polyhedron $\hat{D}$ by its only free edge. Indeed, the link $L$ of the 0 -cell $e_{0}$ of $D$ is homeomorphic to $S^{1} \times \partial I \cup p t \times I$, where $p t \in S^{1}$ (cf. [53, Fig. 5]). Let $\pi: L \rightarrow I$ be the projection. The star $S$ of $e_{0}$ in some triangulation of $D$ is homeomorphic to the cone over $L$, which can be viewed as the mapping cylinder of the constant map $L \rightarrow p t$; we define $\hat{D}$ by replacing $S$ with the mapping cylinder $M C(\pi)$. The target space $I$ of $\pi$ is identified with a free edge $J$ in $\hat{D}$, and clearly $\hat{D}$ is collapsible.

The quotient map $\hat{D} \rightarrow \hat{D} / J=D$, being cell-like, is an $\varepsilon$-homotopy equivalence for each $\varepsilon>0$ by Černavskiı̌'s lemma [23, Lemma 1]; in particular, for each $\varepsilon>0$ there exists an $\varepsilon$-map $f_{\varepsilon}: D \rightarrow \hat{D}$. (Specifically, $f_{\varepsilon}$ is the identity outside $S$, and $\left.f_{\varepsilon}\right|_{S}$ is the composition $S \stackrel{h}{\rightarrow} L \times I \underset{L \times\{1\}=L * \emptyset}{\cup} L * p t \stackrel{g}{\rightarrow} M C(\pi)$, where $h$ is a homeomorphism such that $h^{-1}(L * p t)$ lies in the $\frac{\varepsilon}{2}$-neighborhood of $e_{0}$, and $g$ combines the quotient map $L \times I \rightarrow M C(\pi)$ with a null-homotopy $L * p t \rightarrow$ $I$ of $\pi$.) Since $\hat{D}$ is collapsible, it embeds in a product of two trees ([24]; see Corollary 1.10 above), so $D$ quasi-embeds there; on the other hand, $D$ does not embed in any product of two curves since it is contractible but not collapsible ([24]; see Remark 1.11 above).

As observed in [27, similar arguments show that the Cartesian power $D^{k}$ quasiembeds in a product of $2 k$ trees but does not embed in any product of $2 k$ curves. (This uses the more general result of [24] that no polyhedron $P$ with $\operatorname{rk} H^{1}(P)<n$ and $H^{n}(P, P \backslash\{x\}) \neq 0$ for each $x \in P$ embeds in a product of $n$ curves.) 
Remark 1.19. Zeeman showed that $D \times I$ is collapsible [53], where $D$ is the dunce hat. Hence $D \times I$ embeds in a product of 3 trees by Theorem 1.8. So the absolute retract $X$ in Theorem 1.17 cannot be replaced by $D$. Moreover, it cannot be replaced by any 2-polyhedron $R$, since $R \times I$ embeds in a product of 3 trees by Proposition 1.3 .

Conjecture 1.20. (a) If a compact n-polyhedron $P$ quasi-embeds in a product of $n$ dendrites, then $P \times I$ embeds in a product of $n+1$ trees.

(b) Same if $P$ is a co-locally contractible (see \$5) n-dimensional compactum.

Theorem 1.17 should be compared with the following results.

Theorem 1.21 (Melikhov-Shchepin 36]). (a) If $X$ is a compact $n$-dimensional $A N R$ that quasi-embeds in $I^{2 n-1}, n>3$, then $X \times I$ embeds in $I^{2 n}$.

(b) If $X$ is an acyclic $n$-dimensional compactum, $m>\frac{3(n+1)}{2}$ and $k>0$, then the following are equivalent: (i) $X$ embeds in $I^{m}$; (ii) $X \times I^{k}$ embeds in $I^{m+k}$; (iii) $X \times T^{k}$ embeds in $I^{m+2 k}$, where $T$ denotes the triod.

In conclusion we note that the proof of nonembeddability in Theorem 1.17 involves the same kinds of local geometry and local algebra as that in the following.

Theorem 1.22 (Melikhov-Shchepin [36]). For each $n>1$ there exists a compact $n$-dimensional ANR, quasi-embeddable but not embeddable in $I^{2 n}$.

\section{Collapsible polyhedra}

We use the following combinatorial notation [35, Chapter 2]. Given a poset $P$ and a $\sigma \in P$, the cone $\lceil\sigma\rceil$ is the subposet of all $\tau \in P$ such that $\tau \leq \sigma$, and the dual cone $\lfloor\sigma\rfloor$ is the subposet of all $\tau \in P$ such that $\tau \geq \sigma$. The $\operatorname{link} \operatorname{lk}(\sigma, P)$ is the subposet of all $\tau \in P$ such that $\tau>\sigma$, and the $\operatorname{star} \operatorname{st}(\sigma, P)$ is the subposet of all $\rho \in P$ such that $\rho \leq \tau$ for some $\tau \in\lfloor\sigma\rfloor$. If $K$ is a simplicial complex (viewed as a poset of nonempty faces ordered by inclusion), and $\sigma \in K$, then $\operatorname{lk}(\sigma, K)$ is a simplicial complex, and $\operatorname{st}(\sigma, K)$ is isomorphic to $\lceil\sigma\rceil * \operatorname{lk}(\sigma, K)$ 2

Here the join is defined as follows. The dual cone $C^{*} P$ of the poset $P$ consists of $P$ together with an additional element $\hat{0}$ that is set to be less than every element of $P$. The coboundary $\partial^{*} Q$ of a dual cone $Q=C^{*} P$ is the original poset $P$. (Note the relation with coboundary of cochains.) The product $P \times Q$ of two posets consists of pairs $(p, q)$, where $p \in P$ and $q \in Q$, ordered by $(p, q) \leq\left(p^{\prime}, q^{\prime}\right)$ if $p \leq q$ and $p^{\prime} \leq q^{\prime}$. The join $P * Q=\partial^{*}\left(C^{*} P \times C^{*} Q\right)$. Note that $P * Q=C^{*} P \times Q \cup P \times C^{*} Q$ (union along $P \times Q)$.

The canonical subdivision $P^{\#}$ is the poset of all order intervals of $P$, ordered by inclusion. If $K$ is a simplicial complex, then $\left(C^{*} K\right)^{\#}$ is a cubical complex. Conversely, if $Q$ is a cubical complex and $q \in Q$, then $\operatorname{lk}(q, Q)$ is a simplicial complex, and $\operatorname{st}(q, Q)$ is isomorphic to $\lceil q\rceil \times\left(C^{*} \operatorname{lk}(q, Q)\right)^{\#}$. Moreover, $\operatorname{lk}((p, q), P \times$ $Q)$ is isomorphic to $\operatorname{lk}(p, P) * \operatorname{lk}(q, Q)$. The details can be found in [35, Chapter 2].

Theorem 1.8 now follows from

\footnotetext{
${ }^{2}$ Our $\operatorname{lk}(\sigma, P)$ is a standard notion of link in modern topological combinatorics; we shall need it when $P$ is a cubical complex (where every cone is isomorphic to the poset of nonempty faces of a cube). The notion of link in combinatorial topology of the 1960s was something slightly different: being defined only when $P$ is a simplicial complex, it is canonically isomorphic to our $\operatorname{lk}(\sigma, P)$ but is not identical with it.
} 
Lemma 2.1. Let $K \searrow L$ be a simplicial collapse of simplicial complexes and let $T_{1}, \ldots, T_{n}$ be trees, so that $T=T_{1} \times \ldots \times T_{n}$ is a cubical complex. Suppose that $f:|L| \rightarrow|T|$ is a PL embedding such that $f(|\sigma|)$ is cubulated by a subcomplex of $T$ for every simplex $\sigma$ of $L$. Then each $T_{i}$ embeds in a larger tree $\tilde{T}_{i}$ and $f$ extends to a PL embedding $\bar{f}:|K| \rightarrow|\tilde{T}|$, where $\tilde{T}=\tilde{T}_{1} \times \cdots \times \tilde{T}_{i}$, such that $\bar{f}(|\sigma|)$ is cubulated by a subcomplex of $\tilde{T}$ for every simplex $\sigma$ of $K$.

Moreover, $|T| \cap \bar{f}(|K|)=f(|L|)$, and $|\tilde{T}|$ collapses onto $|T| \cup \bar{f}(|K|)$.

Proof. Arguing by induction, we may assume that $K \searrow L$ is an elementary simplicial collapse. Let $Q$ denote the subcomplex of $T$ cubulating $f(|L|)$, and let $B$ be the subcomplex of $Q$ cubulating the image of the topological frontier of $|L|$ in $|K|$. We may now forget $K, L$ and $f$, remembering only that $|B|$ is a PL ball of some dimension $k<n$. We thus want to construct trees $\tilde{T}_{i} \supset T_{i}$ and a subcomplex $\beta$ of $\tilde{T}_{1} \times \cdots \times \tilde{T}_{n}$ such that $\beta \cap Q=B$ and $|\beta|$ is a PL $(k+1)$-ball.

The boundary of $|B|$ is cubulated by a subcomplex $\partial B$ of $B$. Given a face $q=q_{1} \times \cdots \times q_{n}$ of $B \backslash \partial B$, we have $\operatorname{lk}(q, T) \simeq \operatorname{lk}\left(q_{1}, T_{1}\right) * \cdots * \operatorname{lk}\left(q_{n}, T_{n}\right)$. Each $q_{i}$ is either a vertex or an edge, and then $\operatorname{lk}\left(q_{i}, T_{i}\right)$ is either a finite set or the empty set, accordingly. Let $C$ be set of those $i$ for which $q_{i}$ is a vertex. Then the cube $\lceil q\rceil$ is of dimension $n-\# C$, and consequently the dimension $d-1$ of $\operatorname{lk}(q, B)$ equals $k-n+\# C-1<\# C-1$.

Every vertex $v$ of $\operatorname{lk}(q, T)$ lies in $\operatorname{lk}\left(q_{i}, T_{i}\right)$ for some $i \in C$; in that case let us color $v$ by the $i$ th color. In particular, the subcomplexes $\Lambda:=\operatorname{lk}(q, Q)$ and $S:=\operatorname{lk}(q, B)$ of $\operatorname{lk}(q, T)$ are $C$-colored. Since $\# C>d$, by the Fisk-IzmestievWitte lemma, the $C$-colored combinatorial $(d-1)$-sphere $S$ bounds (abstractly) a $C$-colored combinatorial ball $D$. Let $\Lambda^{+}$be the amalgamated union $\Lambda \cup_{S} D$, that is, the pushout of the diagram $\Lambda \supset S \subset D$ in the category of $C$-colored simplicial complexes and color-preserving simplicial maps.

If $D \backslash S$ contains $k_{i}$ vertices of color $i$, we define a new tree $T_{i}^{+}=T_{i} \cup\left(q_{i} *\left[k_{i}\right]\right)$ by attaching $k_{i}$ new edges to $T_{i}$ at the vertex $q_{i}$ for each $i \in C$ (note that $\left[k_{i}\right]=\emptyset$ and so $T_{i}^{+}=T_{i}$ for each $\left.i \notin C\right)$. Let $T^{+}=T_{1}^{+} \times \cdots \times T_{n}^{+}$. The $C$-coloring of the vertices of $\operatorname{lk}(q, T)$ extends to the similarly defined $C$-coloring of the vertices of $\operatorname{lk}\left(q, T^{+}\right)$. Then any color-preserving identification of the vertices of $D \backslash S$ with the vertices of $\operatorname{lk}\left(q, T^{+}\right)$that are not in $\operatorname{lk}(q, T)$ extends uniquely to a color-preserving simplicial map $\Lambda^{+} \rightarrow \operatorname{lk}\left(q, T^{+}\right)$that extends the inclusion $\Lambda \subset \operatorname{lk}(q, T)$. This simplicial map is injective on vertices, hence is an embedding. By construction, $\Lambda^{+} \cap \operatorname{lk}(q, T)=\Lambda$.

In particular, $D$ is now identified with a subcomplex of $\operatorname{lk}(q, T)$; hence $E:=$ $\lceil q\rceil \times\left(C^{*} D\right)^{\#}$ and $F:=\lceil q\rceil \times D^{\#} \cup(\partial\lceil q\rceil) \times\left(C^{*} D\right)^{\#}$ are identified with subcomplexes of $\lceil q\rceil \times\left(C^{*} \operatorname{lk}\left(q, T^{+}\right)\right)^{\#}=\operatorname{st}\left(q, T^{+}\right)$. Since $D \cap \Lambda=S$, we have $E \cap Q=E \cap B$. Let $Q^{+}=Q \cup E$. Note that $E \cap B$ is the cubical combinatorial $k$-ball $\operatorname{st}(q, B)=$ $\lceil q\rceil \times\left(C^{*} \operatorname{lk}(q, B)\right)^{\#}$, and $F \cap B$ is its boundary, the cubical combinatorial sphere $\partial \operatorname{st}(q, B)=\lceil q\rceil \times \operatorname{lk}(q, B)^{\#} \cup(\partial\lceil q\rceil) \times\left(C^{*} \operatorname{lk}(q, B)\right)^{\#}$. Further note that $\operatorname{st}(q, B) \backslash$ $\partial \operatorname{st}(q, B)$ is the dual cone $\lfloor q\rfloor$ of $q$ in $B$. Then $B^{+}=(B \backslash\lfloor q\rfloor) \cup F$ is a cubical combinatorial $k$-ball, which does not contain $q$.

Since $\Lambda^{+} \cap \operatorname{lk}(q, T)=\Lambda$, we have $Q^{+} \cap T=Q$. Furthermore, $\left|T^{+}\right|$collapses onto $\left|T \cup\left(q_{1} *\left[k_{1}\right]\right) \times \cdots \times\left(q_{n} *\left[k_{n}\right]\right)\right|$ (using conewise collapses of the form $X \times C Y \searrow$ $X \cup Z \times C Y$ where $Z$ is a closed subpolyhedron of $X$ ), which in turn collapses onto $|T \cup E|=\left|T \cup Q^{+}\right|$(using the collapse of the cone $\left|\prod_{i \in C} q_{i} *\left[k_{i}\right]\right|$ onto its subcone $\left.\left|\left(C^{*} D\right)^{\#}\right|\right)$. 
In order to fit the above process in an inductive argument, let us now write $Q_{0}$, $B_{0}$ for the given $Q, B$. Assuming that $Q_{i}, B_{i}$ have been constructed, along with some distinct $q_{1}, \ldots, q_{i} \in\left(B_{0} \backslash \partial B_{0}\right) \backslash B_{i}$, we repeat the above process with $Q=Q_{i}$ and $B=B_{i}$, with one modification: $q$ is now not an arbitrary face of $B_{i} \backslash \partial B_{i}$, but one that is also a face of the original $B_{0} \backslash \partial B_{0}$. Since $q$ is still required to be a face of $B_{i}$, our hypothesis entails that $q \notin\left\{q_{1}, \ldots, q_{i}\right\}$. We set $Q_{i+1}=Q^{+}, B_{i+1}=B^{+}$, and $q_{i+1}=q$. Then $q_{0}, \ldots, q_{i+1} \in\left(B_{0} \backslash \partial B_{0}\right) \backslash B_{i+1}$, which completes the inductive step. Since $B_{0} \backslash \partial B_{0}$ is finite, the number of steps is bounded. If the final step is $r$ th, it is easy to see that $B_{r} \cap B_{0}=\partial B_{0}=\partial B_{r}$, and $B_{0} \cup B_{r}$ bounds a cubical combinatorial $(k+1)$-ball $\beta$ (namely, $\beta$ is the union of all the $(k+1)$ )-balls of the form $E$ ) such that $\beta \cap Q_{0}=B_{0}$ and $\beta \cup Q_{0}=Q_{r}$.

Remark 2.2. The combinatorial type of the ball $\beta$ depends on the order in which $q_{1}, \ldots, q_{r}$ are picked out of $B_{0} \backslash \partial B_{0}$. For instance, suppose that $n=2, k=1$ and the $\operatorname{arc} B_{0}$ consists of $e$ edges (and hence $e+1$ vertices). If $e>1$, then we may take $q_{1}, \ldots, q_{r}$ to be all the nonboundary vertices, ordered consecutively, which will lead to the same $\beta$ as in 24. For instance if $e=2($ so $r=1)$ and $T_{1}=Q_{0}=B_{0}$, $T_{2}=p t$, then $\tilde{T}_{1}=T_{1}, \tilde{T}_{2}$ is a single edge, and $Q_{r}=B_{r}=\tilde{T}_{1} \times \tilde{T}_{2}$ (which amounts to two squares). On the other hand, if we first pick out all the edges (in any order) and then the $e-1$ nonboundary vertices (in any order), the result will be unique but quite different from the above. For instance if $e=2$ (so $r=3$ ) and $T_{1}=Q_{0}=B_{0}$, $T_{2}=p t$, then at the final step $\tilde{T}_{1}$ is a triod, $\tilde{T}_{2}$ contains two edges, and $B_{r}$ consists of four squares. Picking out only vertices but not consecutively may also lead to a $\beta$ different from that in 24 .

Remark 2.3. As discussed in the previous remark, the construction in the proof of Lemma 2.1 depends on the choices of the cubes $q_{1}, \ldots, q_{r}$. Let us describe a canonical range of choices that all lead to the same embedding. Each tree $T_{i}$ is constructed in stages $p t=T_{i 0} \subset \cdots \subset T_{i s}=T_{i}$. The vertices of $T_{i}$ are partially ordered by $v<w$ if there exists a $k<s$ such that $v \in T_{i k}$ and $w \notin T_{i k}$, yet $w$ and $v$ belong to the same component of $\left|T_{i} \backslash\left\lfloor T_{i, k-1}\right\rfloor\right|$. (In particular, incomparable vertices are nonadjacent in the tree.) This yields a partial order on the vertices of $B \backslash \partial B \subset Q \subset T_{1} \times \cdots \times T_{n}$. Let $q_{1}, \ldots, q_{r}$ be the vertices of $B \backslash \partial B$ arranged in some total order extending the constructed partial order. It is clear then that $r$ is indeed the last stage of the construction and that $Q_{r}$ does not depend on the choice of the total order.

An alternative proof of the implication (iii) $\Rightarrow(\mathrm{i})$ in Corollary 1.9 is given by Lemma 2.1 along with the following lemma (take $k=n-1$ ).

Lemma 2.4. Let $L$ be a $k$-dimensional simplicial complex. Then there exist trees $T_{0}, \ldots, T_{k}$ and a PL embedding $f:|L| \rightarrow|T|$, where $T=T_{0} \times \cdots \times T_{k}$ such that $f(|\sigma|)$ is cubulated by a subcomplex of $T$ for every simplex $\sigma$ of $L$.

The prejoin $P+Q$ consists of the elements of $P \cup Q$ with the order $\preceq$ defined as follows: $p \preceq q$ iff either $p, q \in P$ and $p \leq q$ in $P$ or $p, q \in Q$ and $p \leq q$ in $Q$ or $p \in P$ and $q \in Q$. Note that $C^{*} P \simeq p t+P$. It is easy to see that $(P+Q)^{b} \simeq P^{b}+Q^{b}$, where $P^{b}$ denotes the barycentric subdivision (see details in [35]). 
Proof. Let $S_{i}$ be the set of $i$-dimensional simplices of $L$. Then $L$ is a subcomplex of $S_{0}+\cdots+S_{k}$. Hence $L^{\mathrm{b}}$ is a subcomplex of $\left(S_{0}+\cdots+S_{k}\right)^{\mathrm{b}} \simeq S_{0} * \cdots * S_{k}$, which in turn is a subcomplex of $C^{*}\left(S_{0} * \cdots * S_{k}\right)$. Therefore $\left(L^{b}\right)^{\#}$ is a subcomplex of $\left(C^{*}\left(S_{0} * \cdots * S_{k}\right)\right)^{\#} \simeq\left(C^{*} S_{0}\right)^{\#} \times \cdots \times\left(C^{*} S_{k}\right)^{\#}$. Each $\left(C^{*} S_{i}\right)^{\#}$ is a tree, and the assertion follows.

\section{LOCAL COHOMOLOGY}

By $H^{*}$ we denote the Alexander-Spanier cohomology [45, 31, or equivalently (see [44) sheaf cohomology with constant coefficients [9]. If the coefficients are omitted, they are understood to be integer. The case of coefficients in a field is much easier (see [50]) but will not suffice for our purposes.

If $(X, Y)$ is a pair of compacta, $H^{i}(X, Y)$ is isomorphic to the direct limit $\lim H^{i}\left(P_{j}, Q_{j}\right)$, where $\cdots \rightarrow\left(P_{1}, Q_{1}\right) \rightarrow\left(P_{0}, Q_{0}\right)$ is any inverse sequence of pairs of compact polyhedra with inverse limit $(X, Y)$. In particular, every cohomology group $H^{i}(Y, X)$ is countable.

More generally, when $Y$ is closed in $X$ (which we always assume to be metrizable), then $H^{i}(X, Y)$ coincides (see 44]) with the Čech cohomology of $(X, Y)$, which may be defined as the direct limit of the $i$ th cohomology groups of the nerves of all open coverings of $(X, Y)$. In particular, if $Y$ is closed in $X$ and $X$ is $n$-dimensional, then $H^{i}(X, Y)=0$ for $i>n$ (since covers with at most $n$-dimensional nerves form a cofinal subset in the directed set of all open covers of $X$ ).

If $X$ is a compactum and $x \in X$, the local cohomology group $H^{i}(X, X \backslash\{x\})$ is isomorphic to $\lim H^{i-1}\left(U_{i} \backslash\{x\}\right)$, where $U_{0} \supset U_{1} \supset \ldots$ are neighborhoods of $x$ in $X$ such that $\cap \vec{U}_{k}=\{x\}$ and each Int $U_{k} \supset \mathrm{Cl} U_{k+1}$. As observed in [43, §1], this follows from the exact sequences of the pairs $\left(U_{k}, U_{k} \backslash\{x\}\right)$ and the fact that the direct limit functor preserves exactness of sequences. However, this isomorphism will not be used in the sequel.

Instead, we shall use the following more geometric description of the local cohomology groups (parallel to [38, proof of Lemma 1]).

Proposition 3.1. Let $X$ be a compactum, let $x \in X$ and let $U_{1} \supset U_{2} \supset \ldots$ be neighborhoods of $x$ in $X$ such that $\bigcap U_{k}=\{x\}$ and each $\operatorname{Int} U_{k} \supset \mathrm{Cl} U_{k+1}$. Then

$$
H^{i}(X, X \backslash\{x\}) \simeq H^{i}\left(X \times[0, \infty), X \times[0, \infty) \backslash U_{[0, \infty)}\right),
$$

where $U_{[0, \infty)}=U_{0} \times[0,1) \cup U_{1} \times[1,2) \cup U_{2} \times[2,3) \cup \ldots$

Note that if the $U_{k}$ are open, then $X \times[0, \infty) \backslash U_{[0, \infty)}$ is a closed subset of $X \times[0, \infty)$. Hence from the preceding discussion we obtain

Corollary 3.2. If $X$ is an $n$-dimensional compactum, $H^{i}(X, X \backslash\{x\})=0$ for $i>n+1$ and all $x \in X$.

Proof of Proposition 3.1. We shall show that $(X, X \backslash\{x\})$ is "almost" homotopy equivalent to the mapping telescope of pairs $\left(X, X \backslash U_{i}\right)$, meaning that there is a map of pairs in one direction, which admits a homotopy inverse separately on each entry of the pair; by the Five Lemma, this is just good enough as long as cohomology is concerned.

The projection $X \times[0, \infty) \rightarrow X$ yields a map of pairs $f:(X \times[0, \infty), X \times$ $\left.[0, \infty) \backslash U_{[0, \infty)}\right) \rightarrow(X, X \backslash\{x\})$. If $\varphi: X \backslash\{x\} \rightarrow[0, \infty)$ is a map such that $\varphi^{-1}([0, n]) \subset X \backslash U_{n}$, then $g: X \backslash\{x\} \rightarrow X \times[0, \infty)$ defined by $g(y)=(y, \varphi(y))$ is 
an embedding into $X \times[0, \infty) \backslash U_{[0, \infty)}$. It is easy to see that $g$ is homotopy inverse to the restriction $h: X \times[0, \infty) \backslash U_{[0, \infty)} \rightarrow X \backslash\{x\}$ of the projection $X \times[0, \infty) \rightarrow X$; hence $h$ is a homotopy equivalence. Using the isomorphisms induced by $g$ and the homotopy equivalence $X \times[0, \infty) \rightarrow X$, the Five Lemma implies that $f^{*}$ is an isomorphism.

By well-known arguments (see [37, proof of Theorem 4] or [34, proof of equation (*) in $\S 1$.B or proof of Theorem 3.1(b)]), Proposition 3.1 gives rise to a Milnor-type natural short exact sequence (found explicitly in [18]):

$$
0 \rightarrow \lim _{\leftarrow}^{1} H^{i-1}\left(X, X \backslash U_{k}\right) \rightarrow H^{i}(X, X \backslash\{x\}) \rightarrow \lim _{\leftarrow} H^{i}\left(X, X \backslash U_{k}\right) \rightarrow 0 .
$$

In particular,

$$
H^{n+1}(X, X \backslash\{x\}) \simeq \lim _{\leftarrow}^{1} H^{n}\left(X, X \backslash U_{k}\right),
$$

if $X$ is an $n$-dimensional compactum.

Lemma 3.3. If $X$ and $Y$ are compacta of dimensions $n$ and $m$, and $x \in X$ and $y \in Y$ are such that $H^{n+1}(X, X \backslash\{x\})=0$ and $H^{m+1}(Y, Y \backslash\{y\})=0$, then also $H^{n+m+1}(X \times Y, X \times Y \backslash\{(x, y)\})=0$.

Proof. Since cohomology groups of pairs of compacta are countable, the hypothesis and the conclusion can be reformulated in terms of the Mittag-Leffler condition, using the isomorphism $(*)$ and Gray's Lemma (see [34, Lemma 3.3]). Then the assertion follows (cf. [36, proof of Lemma 3.6(b)]) from the naturality in the Künneth formula [9, Theorem II.15.2 and Proposition II.12.3] (see also [31, Theorem 7.1], which implies the relative case using the map excision axiom).

Lemma 3.4. If $X$ is an n-dimensional compactum and $H^{n+1}(X, X \backslash\{x\})=0$, then $H^{n+1}(Y, Y \backslash\{x\})=0$ for every $n$-dimensional compactum $Y \subset X$ containing $x$.

Proof. Let $U_{k}$ be open neighborhoods of $x$ in $X$ as in Proposition 3.1. The restriction map $H^{n}\left(X, X \backslash U_{k}\right) \stackrel{f_{k}}{\longrightarrow} H^{n}\left(Y, Y \backslash U_{k}\right)$ is onto from the exact sequence of the triple $\left(X, Y \cup\left(X \backslash U_{k}\right), X \backslash U_{k}\right)$, due to $H^{n+1}\left(X, Y \cup\left(X \backslash U_{k}\right)\right)=0$. Then $\lim _{\leftarrow}^{1} f_{k}$ is onto from the six-term exact sequence of inverse and derived limits (see [34, Theorem 3.1(d)] for a geometric proof) associated to the short exact sequences

$$
0 \rightarrow \operatorname{ker} f_{k} \rightarrow H^{n}\left(X, X \backslash U_{k}\right) \stackrel{f_{k}}{\longrightarrow} H^{n}\left(Y, Y \backslash U_{k}\right) \rightarrow 0 .
$$

But by naturality of the isomorphism $(*), \lim _{\leftarrow}^{1} f_{k}$ is identified with the restriction map $H^{n+1}(X, X \backslash\{x\}) \rightarrow H^{n+1}(Y, Y \backslash\{y\})$.

Remark 3.5. The Menger curve $M$ contains points $x$ such that $H^{2}(M, M \backslash\{x\}) \neq 0$. (Since $M$ is known to be homogeneous, this applies to every $x \in M$.) For let $Y$ be the subspace $\mathbb{N}^{+} \times[0,1) \cup[0, \infty] \times\{1\}$ of $[0, \infty] \times[0,1]$, where $\mathbb{N}^{+}=\{0,1, \ldots, \infty\}$, and let $y=(\infty, 1) \in Y$. Let us represent $Y \backslash\{y\}$ as a union $\bigcup K_{i}$, where each $K_{i}$ is compact and lies in Int $K_{i+1}$ (and not just in $\left.K_{i+1}\right)$. Then $\cdots \rightarrow \tilde{H}^{0}\left(K_{1}\right) \rightarrow \tilde{H}^{0}\left(K_{0}\right)$ is of the form $\cdots \rightarrow \bigoplus_{S_{1}} \mathbb{Z} \rightarrow \bigoplus_{S_{0}} \mathbb{Z}$, where $S_{0} \supset S_{1} \supset \ldots$ is a nested sequence of infinite countable sets with $\bigcap S_{i}=\emptyset$. Since $H^{1}(Y)=0=\tilde{H}^{0}(Y)$, the inverse sequence $\cdots \rightarrow H^{1}\left(Y, K_{1}\right) \rightarrow H^{1}\left(Y, K_{0}\right)$ is of the same form. Clearly it does not satisfy the Mittag-Leffler condition and consists of countable groups, so by 
Gray's Lemma (see [34, Lemma 3.3]) its derived limit is nontrivial. (In fact, it is easy to see, similarly to [34, Example 3.2], that $\lim _{\leftarrow}^{1} H^{1}\left(Y, K_{i}\right) \simeq \prod_{\mathbb{N}} \mathbb{Z} / \bigoplus_{\mathbb{N}} \mathbb{Z}$.) Thus by $(*), H^{2}(Y, Y \backslash\{y\}) \neq 0$. Since $Y$ embeds into $M$, Lemma 3.4 implies $H^{2}(M, M \backslash\{x\}) \neq 0$, where $x$ is the image of $y$.

Lemma 3.6. If $X$ is a local dendrite, then $H^{2}(X, X \backslash\{x\})=0$ for every $x \in X$.

The proof is a bit technical; let us explain informally some intuition behind it. There are just two basic examples of inverse sequences of countable abelian groups with nonzero $\lim ^{1}:$ (i) $\ldots \stackrel{p_{1}}{\longrightarrow} \mathbb{Z} \stackrel{p_{0}}{\longrightarrow} \mathbb{Z}$, each $p_{i}$ being a nonzero prime (this occurs

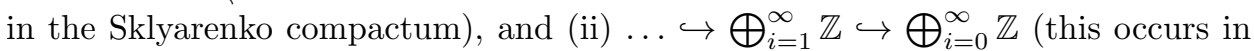
Remark 3.5] and is called "Jacob's ladder" in [20]). Example (i) cannot occur in (*) with $n=1$, because there is "not enough room for twisting" in one-dimensional spaces, so we cannot expect to find even a single multiplication as in (i). On the other hand, if $X$ is an $\mathrm{LC}_{n}$ compactum, then we cannot find example (ii) in (*), because $n$-cohomology of compact subsets of $X$ is "almost" finitely generated in the sense that for every two compact subsets $K \subset X$ and $L \subset$ Int $K$, the image of $H^{n}(K) \rightarrow H^{n}(L)$ is finitely generated [9, II.17.5 and V.12.8].

Proof. Let us represent $X \backslash\{x\}$ as a union $\bigcup K_{i}$, where each $K_{i}$ is compact and lies in Int $K_{i+1}$. Since $X$ is locally contractible, for each $n$ (in particular, for $n=1$ ), each inclusion map $K_{i} \rightarrow K_{i+1}$ factors through a (not necessarily embedded in $X) \mathrm{LC}_{n}$ compactum $L_{i}$ [34, Theorem 6.11]. We recall that $\mathrm{LC}_{n}$ compacta have finitely generated cohomology and (Steenrod) homology in dimensions $\leq n$ (see [9, II.17.7 and V.12.8], 34, 6.11]). Universal coefficients formulas then imply that $\mathrm{LC}_{1}$ compacta have free abelian $H^{1}$ (see [9, V.12.8]) and consequently also free abelian $H_{0}$ (see [9, §V.3, Eq. (9) on p. 292]).

Consider a composition $f: L_{i} \rightarrow K_{i+1} \rightarrow K_{j} \rightarrow L_{j}$. By the naturality of the universal coefficients formula (see [9, V.12.8, V.13.7]), $f^{*}: H^{0}\left(L_{j}\right) \rightarrow H^{0}\left(L_{i}\right)$ is dual to $f_{*}: H_{0}\left(L_{i}\right) \rightarrow H_{0}\left(L_{j}\right)$. The image of $f_{*}$ is a subgroup of the free abelian group $H_{0}\left(L_{j}\right)$. So it is itself free abelian, in particular, projective as a $\mathbb{Z}$-module. Hence $f_{*}$ is a split epimorphism onto its image. Then the inclusion of the image of $f^{*}$ into $H^{0}\left(L_{i}\right)$ is a split monomorphism. (Indeed, given abelian group homomorphisms $f_{*}: G \rightarrow H, f^{*}: \operatorname{Hom}(H, \mathbb{Z}) \rightarrow \operatorname{Hom}(G, \mathbb{Z})$ defined by $f^{*}(\psi)=\psi f_{*}$, and $s: \operatorname{im} f_{*} \rightarrow G$ such that $f_{*} s f_{*}=f_{*}$, define $r: \operatorname{Hom}(G, \mathbb{Z}) \rightarrow \operatorname{im} f^{*}$ by $r(\varphi)=\varphi s f_{*}$; then $r f^{*}=f^{*}$, i.e. $r\left(\psi f_{*}\right)=\psi f_{*}$ for each $\psi \in \operatorname{Hom}(H, \mathbb{Z})$.) Thus $f^{*}$ is a homomorphism onto a direct summand of $H^{0}\left(L_{i}\right)$. The finitely generated group $H^{0}\left(L_{i}\right)$ contains no infinitely decreasing chain of direct summands; so the inverse sequence $\cdots \rightarrow H^{0}\left(L_{1}\right) \rightarrow H^{0}\left(L_{0}\right)$ satisfies the Mittag-Leffler condition; hence so does $\cdots \rightarrow H^{0}\left(K_{1}\right) \rightarrow H^{0}\left(K_{0}\right)$.

On the other hand, consider a composition $g: L_{i} \rightarrow K_{i+1} \rightarrow X$. The image of $g^{*}: H^{1}(X) \rightarrow H^{1}\left(L_{i}\right)$ is a subgroup of the free abelian group $H^{1}\left(L_{i}\right)$. So it is itself free abelian, in particular, projective as a $\mathbb{Z}$-module. Hence $g^{*}$ is a split epimorphism onto its image. Then the kernel of $g^{*}$ is a direct summand in $H^{1}(X)$. The finitely generated group $H^{1}(X)$ contains no infinitely decreasing chain of direct summands; hence the homomorphisms $H^{1}(X) \rightarrow H^{1}\left(L_{i}\right)$ have the same kernel for all sufficiently large $i$. Then so also do the homomorphisms $H^{1}(X) \rightarrow H^{1}\left(K_{i}\right)$. 
Since $X$ is 1-dimensional, the latter are surjective. Hence $H^{1}\left(K_{i+1}\right) \rightarrow H^{1}\left(K_{i}\right)$ are isomorphisms for sufficiently large $i$. In particular, $\cdots \rightarrow H^{1}\left(K_{1}\right) \rightarrow H^{1}\left(K_{0}\right)$ satisfies the dual Mittag-Leffler condition.

Thus by Dydak's Lemma (see [34, Lemma 3.11]), $\cdots \rightarrow H^{1}\left(X, K_{1}\right) \rightarrow H^{1}\left(X, K_{0}\right)$ satisfies the Mittag-Leffler condition. Hence $\lim _{\leftarrow}^{1} H^{1}\left(X, K_{i}\right)=0$, and the assertion follows from $(*)$.

\section{Sklyarenko's COMPACTUM}

We note that if the compactum $X$ is the limit of an inverse sequence of compacta $X_{i}$, all of which embed in $Y$, then $X$ quasi-embeds in $Y$ (for it follows from the definition of the topology of the inverse limit that the maps $X \stackrel{p_{i}^{\infty}}{\longrightarrow} X_{i} \subset Y$ are $\varepsilon_{i}$-maps with respect to any fixed metric on $X$, where $\varepsilon_{i} \rightarrow 0$ as $\left.i \rightarrow \infty\right)$. The converse implication (which we shall not need here) holds when $Y$ is a polyhedron (a simple proof should appear in a future version of [36]; see also [33, Theorem 1] but beware that their " $\varepsilon$-maps" are required to be surjective).

4.1 (Sklyarenko's compactum). Given a direct sequence $X_{1} \rightarrow X_{2} \rightarrow \ldots$, the mapping telescope $\operatorname{Tel}\left(X_{1} \rightarrow X_{2} \rightarrow \ldots\right)$ is the infinite union $M C\left(X_{1} \rightarrow X_{2}\right) \cup_{X_{2}}$ $M C\left(X_{2} \rightarrow X_{3}\right) \cup_{X_{3}} \ldots$ of the mapping cylinders (the direct limit of the finite unions). Let $X$ be the one-point compactification of the mapping telescope of the direct sequence

$$
S^{1} \stackrel{2}{\rightarrow} S^{1} \stackrel{2}{\rightarrow} \ldots
$$

of two-fold coverings. It is easy to see that $X$ is a contractible and locally contractible 2-dimensional compactum, and so an AR. It was introduced by Je. G. Sklyarenko [43, Example 4.6]. We shall call $X$ the Sklyarenko compactum.

Proposition 4.2. Sklyarenko's compactum quasi-embeds in a product of two dendrites.

Proof. Let us represent $X$ as an inverse limit of polyhedra. To this end, consider the following mapping telescope of a direct sequence:

$$
X_{i}=\operatorname{Tel}\left(S_{1}^{1} \stackrel{2}{\rightarrow} \ldots \stackrel{2}{\rightarrow} S_{i}^{1} \rightarrow p t\right),
$$

where each $S_{j}^{1}$ stands for a copy of $S^{1}$. Note that $X$ contains the cone $D^{2}=$ $\operatorname{Tel}\left(S_{i}^{1} \rightarrow p t\right)$. Let $f_{i}: X_{i+1} \rightarrow X_{i}$ be the composition of the quotient map $X_{i+1} \rightarrow$ $X_{i+1} / D^{2}$ and a homeomorphism $X_{i+1} / D^{2} \rightarrow X_{i}$ which is the identity on $\operatorname{Tel}\left(S_{1}^{1} \stackrel{2}{\rightarrow}\right.$ $\ldots \stackrel{2}{\rightarrow} S_{i}^{1}$ ). Then $X$ is homeomorphic to the inverse limit of $\ldots \stackrel{f_{2}}{\longrightarrow} X_{2} \stackrel{f_{1}}{\longrightarrow} X_{1}$.

Notice that each $X_{i}$ is a collapsible 2-polyhedron. Hence by a result of Koyama, Krasinkiewicz and Spież (see Corollary 1.10), $X_{i}$ embeds in a product of two trees $T_{i}$ and $T_{i}^{\prime}$. Let us consider the cluster $T=\lim _{\leftarrow}\left(\cdots \rightarrow T_{1} \vee T_{2} \vee T_{3} \rightarrow T_{1} \vee T_{2} \rightarrow T_{1}\right)$ of the $T_{i}$, where the basepoint of each $T_{i}$ is one of its endpoints. Let $T^{\prime}$ be the analogous cluster of the trees $T_{i}^{\prime}$. Then $T$ and $T^{\prime}$ are dendrites, $T$ contains a copy of each $T_{i}$, and $T^{\prime}$ contains a copy of each $T_{i}^{\prime}$. Thus each $X_{i}$ embeds in $T \times T^{\prime}$. Therefore $X$ quasi-embeds there.

Let $X$ be the Sklyarenko compactum and let $\infty \in X$ denote the remainder point of the one-point compactification. It is easy to see that $H^{3}(X, X \backslash\{\infty\})$ is nonzero 43 . More generally, let us compute $H^{3+k}\left(X \times I^{k}, X \times I^{k} \backslash\{(\infty, 0)\}\right)$, where 
$I=[-1,1]$. Let $F_{i}$ be the union of the first $i$ mapping cylinders in the mapping telescope:

$$
F_{i}=\operatorname{Tel}\left(S_{1}^{1} \stackrel{2}{\rightarrow} \ldots \stackrel{2}{\rightarrow} S_{i}^{1}\right) .
$$

Each $F_{i}$ collapses onto $S_{i}^{1}$, and these collapses identify up to homotopy the inclusions $F_{i} \subset F_{i+1}$ with the two-fold coverings $S_{i}^{1} \stackrel{2}{\rightarrow} S_{i+1}^{1}$. Hence the inverse sequence $\cdots \rightarrow H^{1}\left(F_{2}\right) \rightarrow H^{1}\left(F_{1}\right)$ is of the form $\ldots \stackrel{2}{\rightarrow} \mathbb{Z} \stackrel{2}{\rightarrow} \mathbb{Z}$. Since $X$ is an $\mathrm{AR}$, so is the inverse sequence $\cdots \rightarrow H^{2}\left(X, F_{2}\right) \rightarrow H^{2}\left(X, F_{1}\right)$. Let $G_{i}=$ $F_{i} \times I^{k} \cup X \times\left(I^{k} \backslash\left(-\frac{1}{i}, \frac{1}{i}\right)^{k}\right)$. By the Künneth formula (see references in the proof of Lemma 3.3),$H^{2+k}\left(X \times I^{k}, G_{i}\right) \simeq H^{2}\left(X, F_{i}\right)$, and the inverse sequence $\cdots \rightarrow H^{2+k}\left(X \times I^{k}, G_{2}\right) \rightarrow H^{2+k}\left(X \times I^{k}, G_{1}\right)$ is again of the form $\ldots \stackrel{2}{\rightarrow} \mathbb{Z} \stackrel{2}{\rightarrow} \mathbb{Z}$. In particular, it does not satisfy the Mittag-Leffler condition, so by Gray's Lemma (see [34, Lemma 3.3]) its derived limit is nontrivial. (In fact, it is easy to compute that it is isomorphic to $\mathbb{Z}_{2} / \mathbb{Z}$, where $\mathbb{Z}_{2}$ is the group of 2-adic integers; see [34, Example 3.2].) Thus by $(*), H^{3+k}\left(X \times I^{k}, X \times I^{k} \backslash\{(\infty, 0)\}\right) \neq 0$.

Theorem 4.3. If $X$ is the Sklyarenko compactum, $X \times I^{k}$ does not embed in any product of $2+k$ local dendrites.

Proof. Suppose $X \times I^{k} \subset Y_{1} \times \cdots \times Y_{n}$, where the $Y_{i}$ are local dendrites. Then $(\infty, 0) \in X \times I^{k}$ is of the form $\left(y_{1}, \ldots, y_{n}\right)$. By Lemma 3.6, $H^{2}\left(Y_{i}, Y_{i} \backslash\left\{y_{i}\right\}\right)=0$ for each $i$. Then by Lemma 3.3. $H^{3+k}\left(\prod Y_{i}, \prod Y_{i} \backslash\left\{\left(y_{i}\right)\right\}\right)=0$. Therefore by Lemma 3.4. $H^{3+k}\left(X \times I^{k}, X \times I^{k} \backslash\{(\infty, 0)\}\right)=0$. This contradicts the above computation.

Theorem 4.4 (Koyama-Krasinkiewicz-Spież). If a compact $n$-dimensional ANR embeds in a product of $n$ curves, then it embeds in a product of $n$ local dendrites.

Proof. It is well-known that locally contractible compacta have finitely generated cohomology groups (see [9, II.17.7], [34, 6.11]). If a locally connected $n$-dimensional compactum $X$ with finitely generated $H^{n}(X)$ embeds in a product of $n$ curves, then the first several lines of the proof of Theorem 2.B.1 in 24] (which contain further references) produce an embedding of $X$ in a product of $n$ local dendrites.

Theorems 4.3 and 4.4 have the following

Corollary 4.5. Sklyarenko's compactum multiplied by $I^{k}$ does not embed in any product of $2+k$ curves.

Corollary 4.5 combines with Proposition 4.2 to imply Theorem 1.17

Remark 4.6. If $\cdots \rightarrow G_{1} \rightarrow G_{0}$ is an inverse sequence of countable groups, let $\lim _{\leftarrow}^{1}{ }_{f g} G_{i}$ be the direct $\operatorname{limit} \lim _{\rightarrow} L_{\alpha}$ of the derived limits $L_{\alpha}=\lim _{\leftarrow}{ }^{1} H_{\alpha i}$ over all inverse sequences $\cdots \rightarrow H_{\alpha 1} \rightarrow H_{\alpha 0}$ of finitely generated subgroups $H_{\alpha_{i}} \subset G_{i}$, where the bonding maps are the restrictions of those in $\cdots \rightarrow G_{1} \rightarrow G_{0}$. Some results about $\lim ^{1}{ }_{f g}$ will appear in a future paper by the first author. By using the functor $\lim _{\leftarrow}^{\leftarrow}{ }_{f g}$ in place of $\lim _{\leftarrow}^{1}$, it should be possible to refine the proof of Theorem 4.3 so as to obtain a purely algebraic proof of Corollary 4.5 without using Theorem 4.4 . 
Remark 4.7. The same arguments (only using the general case of Corollary 1.9 rather than the easier 2-dimensional case) show that the $n$-dimensional Sklyarenko compactum (similarly defined with $S^{n-1}$ in place of $S^{1}$ ) quasi-embeds in a product of $n$ dendrites but does not embed in a product of $n$ curves.

\section{Co-LOCAl CONTRACTiBility}

Let us call a compactum $X$ co-locally contractible at $x \in X$ if every neighborhood $U$ of $x$ contains a neighborhood $V$ of $x$ such that the inclusion $X \backslash\{x\} \subset X$ is homotopic to a map $X \backslash\{x\} \rightarrow X \backslash V \subset X$ by a homotopy keeping $X \backslash U$ fixed. (Equivalently, every neighborhood $U$ of $x$ contains a neighborhood $V$ of $x$ such that for every neighborhood $W$ of $x$ contained in $V$, the inclusion $X \backslash$ $W \subset X$ is homotopic to a map $X \backslash W \rightarrow X \backslash V$ by a homotopy keeping $X \backslash U$ fixed.) We call $X$ co-locally contractible if it is co-locally contractible at every point. (Compare Borsuk's idea of co-localization [5, §IX.16] and the co-local connectedness of Krasinkiewicz and Minc [26].)

Remark 5.1. A slightly stronger property than co-local contractibility, obtained by replacing the inclusion $X \backslash\{x\} \subset X$ with the identity map of $X \backslash\{x\}$, is known as reverse (or backward) tameness of $X \backslash\{x\}$ (see [41, [20]). Dually, $X \backslash\{x\}$ is called forward tame if there exists a closed neighborhood $U$ of $x$ such that for every neighborhood $V$ of $x$, the inclusion $V \backslash\{x\} \subset X \backslash\{x\}$ is properly homotopic to a map $V \backslash\{x\} \rightarrow U \backslash\{x\} \subset X \backslash\{x\}$ (see [41], 20]). It is not hard to see (even if it appears surprising) that forward tameness of $X \backslash\{x\}$ implies local contractibility of $X$ at $x$. To see that the converse implication fails, let $P$ be the suspension of a noncontractible acyclic polyhedron and let its basepoint $b$ be one of the two suspension points; alternatively let $P$ be the dunce hat and $b$ its unique 0 -cell. Then the cluster $C=\lim _{\leftarrow}(\cdots \rightarrow P \vee P \vee P \rightarrow P \vee P \rightarrow P)$ of copies of $P$ is an AR, yet it follows from Dydak-Segal-Spież [14] that $C \backslash\{b\}$ is not forward tame.

Proposition 5.2. If an $n$-dimensional compactum $X$ is co-locally contractible at $x$, then $H^{n+1}(X, X \backslash\{x\})=0$.

Proof. This is a straightforward diagram chasing. The hypothesis implies that, with $x, U$ and $V$ as above and for each $i$, the restriction map $H^{i}(X \backslash\{x\}) \rightarrow H^{i}(X \backslash V)$ is a split injection on the image of $H^{i}(X)$. Hence the image of the forgetful map $f: H^{i}(X \backslash\{x\}, X \backslash V) \rightarrow H^{i}(X \backslash\{x\})$ lies in the image of $H^{i}(X)$. The latter equals the kernel of the coboundary map $\delta: H^{i}(X \backslash x) \rightarrow H^{i+1}(X, X \backslash\{x\})$; hence $\delta f=0$. Since this $\delta f: H^{i}(X \backslash\{x\}, X \backslash V) \rightarrow H^{i+1}(X, X \backslash\{x\})$ is also the coboundary map, the restriction $H^{i+1}(X, X \backslash\{x\}) \rightarrow H^{i+1}(X, X \backslash V)$ must be an injection. Finally, since $X$ is $n$-dimensional and without loss of generality $V$ is open, $H^{n+1}(X, X \backslash V)=0$. Thus $H^{n+1}(X, X \backslash\{x\})=0$.

\section{ACKNowledgments}

The authors are grateful to Professors Krasinkiewicz and Spież for posing the problem and for stimulating discussions, and the referee for helpful remarks. The first author also thanks J. Dydak, O. Frolkina and Je. V. Shchepin for relevant conversations. 


\section{REFERENCES}

[1] C. Hog-Angeloni, W. Metzler, and A. Sieradski (eds.), Two-Dimensional Homotopy and Combinatorial Group Theory, London Math. Soc. Lect. Note Ser., vol. 197, Cambridge University Press, 1993. MR1279174 (95g:57006)

[2] T. Banakh and V. Valov, General Position Properties in Fiberwise Geometric Topology (2010). arxiv:1001:2494.

[3] I. Berstein, M. Cohen, and R. Connelly, Contractible, non-collapsible products with cubes, Topology 17 (1978), 183-187. MR0467762 (57:7614)

[4] K. Borsuk, Über das Phänomen der Unzerlegbarkeit in der Polyedertopologie, Comment. Math. Helv. 8 (1935), no. 1, 142-148. MR1509522

[5] Theory of Retracts, Monogr. Mat., vol. 44, Państwowe Wydawnictwo Naukowe, Warszawa, 1967. MR0216473 (35:7306)

[6] _ Remark on the Cartesian product of two $\ell$-dimensional spaces, Bull. Acad. Polon. Sci. Sér. Sci. Math. Astronom. Phys. 23 (1975), no. 9, 971-973. MR0394636 (52:15437)

[7] P. L. Bowers, General position properties satisfied by finite products of dendrites, Trans. Amer. Math. Soc. 288 (1985), no. 2, 739-753. MR776401 (86f:54063)

[8] J. Bracho and L. Montejano, The scorpions: examples in stable noncollapsibility and in geometric category theory, Topology 30 (1991), 541-550. MR.1133871 (93a:57027)

[9] G. E. Bredon, Sheaf Theory, 2nd ed., Grad. Texts in Math., vol. 170, Springer-Verlag, New York, 1997. MR1481706 (98g:55005)

[10] R. P. Carpentier, Extending triangulations of the 2-sphere to the 3-disk preserving a 4coloring. arxiv:1101:5537.

[11] M. M. Cohen, Simplicial structures and transverse cellularity, Ann. Math. 85 (1967), 218245. MR0210143 (35:1037)

[12] _ Whitehead torsion, group extensions, and Zeeman's conjecture in high dimensions, Topology 16 (1977), no. 1, 79-88. MR0436155(55:9105)

[13] M. L. Curtis, On 2-complexes in 4-space, Topology of 3-Manifolds and Related Topics (Proc. Univ. of Georgia Institute, 1961), Prentice-Hall, Englewood Cliffs, N.J., 1962, pp. 204-207. MR0140114 (25:3537)

[14] J. Dydak, J. Segal, and S. Spież, On questions of strict contractibility, Topol. Appl. 120 (2002), no. 1-2, 67-75. MR.1895484 (2003c:55010)

[15] S. Fisk, Variations on coloring, surfaces and higher-dimensional manifolds, Adv. Math. 25 (1977), no. 3, 226-266. MR0454979 (56:13221)

[16] D. Gillman, The Poincaré conjecture is true in the product of any graph with a disk, Proc. Amer. Math. Soc. 110 (1990), 829-834. MR1021898 (91b:57015)

[17] D. Gillman, S. Matveev, and D. Rolfsen, Collapsing and reconstruction of manifolds, Geometric topology (Haifa, 1992), Contemp. Math., vol. 164, Amer. Math. Soc., 1994, pp. 35-39. MR 1282753 (95c:57036)

[18] A. É. Harlap, Local homology and cohomology, homology dimension and generalized manifolds, Mat. Sbornik 96 (1975), no. 3, 347-373; English transl., Math. USSR — Sb. 25 (1975), no. 3, 323-349. MR0645364 (58:31056)

[19] A. Hatcher and N. Wahl, Stabilization for mapping class groups of 3-manifolds, Duke Math. J. 155 (2010), no. 2, 205-269. MR2736166 (2012c:57001)

[20] B. Hughes and A. Ranicki, Ends of Complexes, Cambridge Tracts in Math., vol. 123, 1996. MR 1410261 (98f:57039)

[21] J. R. Isbell, Six theorems about injective metric spaces, Comm. Math. Helv. 39 (1964), 65-76. MR0182949(32:431)

[22] I. Izmestiev, Extension of colorings, European J. Combin. 26 (2005), 779-781. MR.2127696 (2005j:05032)

[23] V. P. Kompaniec and A. V. Černavskiı̌, Equivalence of two classes of sphere mappings, Dokl. Akad. Nauk SSSR 169 (1966), 1266-1268; English transl., Soviet Math. Dokl. 7 (1966), 1083-1085. MR0240788(39:2133)

[24] A. Koyama, J. Krasinkiewicz, and S. Spież, Generalized manifolds in products of curves, Trans. Amer. Math. Soc. 363 (2011), 1509-1532. MR2737275 (2012c:57042)

[25] _ Embedding compacta into products of curves. arxiv:0712:3470v1.

[26] J. Krasinkiewicz and P. Minc, On a notion dual to local connectedness, Proc. Int. Conf. on Geometric Topology, PWN (Polish Sci. Publ.), Warszawa, 1980, pp. 265-267. 
[27] J. Krasinkiewicz and S. Spież, On embedding of the dunce hat, Proc. Amer. Math. Soc. (to appear).

[28] R. Kreher and W. Metzler, Simpliziale Transformationen von Polyedern und die ZeemanVermutung, Topology 22 (1983), 19-26. MR682057 (84e:57022)

[29] W. Kuperberg, On embeddings of manifolds into Cartesian products of compacta, Bull. Acad. Pol. Sci. Ser. Math. 26 (1978), 845-848. MR518991 (81i:57011)

[30] Jie Hua Mai and Yun Tang, An injective metrization for collapsible polyhedra, Proc. Amer. Math. Soc. 88 (1983), no. 2, 333-337. MR695270 (84g:54036)

[31] W. S. Massey, Homology and Cohomology Theory. An Approach Based on Alexander-Spanier Cochains, Mono. and Textbooks in Pure and Appl. Math., vol. 46, Marcel Dekker Inc., New York, 1978. The author's guide for reading this book: How to give an exposition of the Čech-Alexander-Spanier type homology theory, Amer. Math. Monthly 85 (1978), 75-83. MR0488016, MR0488017(58:7595)

[32] S. V. Matveev, Algorithmic Topology and Classification of 3-Manifolds, Springer, 2007; Russian transl. in MCCME, Moscow, 2007. MR2341532

[33] S. Mardešić and J. Segal, e-mappings onto polyhedra, Trans. Amer. Math. Soc. 109 (1963), 146-164. MR0158367 (28:1592)

[34] S. A. Melikhov, Steenrod homotopy, Uspekhi Mat. Nauk 64 (2009), no. 3, 73-166; English transl., Russian Math. Surveys 64 (2009), no. 3, 469-551. MR2553079 (2011d:55022)

[35] , Combinatorics of combinatorial topology. arxiv:1208.6309v1.

[36] S. A. Melikhov and E. V. Shchepin, The telescope approach to embeddability of compacta. arxiv:math.GT/0612085v1.

[37] J. Milnor, On the Steenrod homology theory, London Math. Soc. Lecture Note Ser., vol. 226, Cambridge Univ. Press, Cambridge, 1995, pp. 79-96. MR.1388297 (98d:55005)

[38] _ On axiomatic homology theory, Pacific J. Math. 12 (1962), 337-341. MR.0159327 $(28: 2544)$

[39] J. Nagata, Note on dimension theory for metric spaces, Fund. Math. 45 (1958), 143-181. MR0105081 (21:3827)

[40] L. Pontryagin, A classification of continuous transformations of a complex into a sphere. I, Dokl. Akad. Nauk. SSSR (C. R. Acad. Sci. de l'URSS) 19 (1938), 361-363 (English).

[41] F. Quinn, Lectures on controlled topology: mapping cylinder neighborhoods, Topology of HighDimensional Manifolds, No. 1, 2 (Trieste, 2001), ICTP Lect. Notes, vol. 9, Abdus Salam Int. Cent. Theoret. Phys., Trieste, 2002, pp. 461-489. MR1937021 (2004b:57047)

[42] J. Segal, A. Skopenkov, and S. Spiez, Embeddings of polyhedra in $\mathbf{R}^{m}$ and the deleted product obstruction, Topology Appl. 85 (1998), no. 1-3, 335-344. MR.1617472 (99b:57046)

[43] E. G. Skljarenko, On homologically locally connected spaces, Izv. Akad. Nauk SSSR Ser. Mat. 44 (1980), no. 6, 1417-1433, 1440; English transl., Math. USSR - Izvestiya 17 (1981), no. 3, 601-614. MR603583(82e:55011)

[44] E. G. Sklyarenko, The homology and cohomology of general spaces, Current Problems in Mathematics. Fundamental Directions, Vol. 50, Itogi Nauki i Tekhniki, Akad. Nauk SSSR Vsesoyuz. Inst. Nauchn. i Tekhn. Inform., Moscow, 1989, pp. 129-266; English transl., General Topology II, Encycl. Math. Sci., vol. 50, Springer, 1996, pp. 119-256. MR1004026 (90h:55005) MR 1392482

[45] E. H. Spanier, Algebraic Topology, McGraw-Hill, New York, 1966. MR0210112 (35:1007)

[46] Y. Sternfeld, Linear superpositions with mappings which lower dimension, Trans. Amer. Math. Soc. 277 (1983), no. 2, 529-543. MR694374 (85b:26015)

[47] _ Mappings in dendrites and dimension, Houston J. Math. 19 (1993), no. 3, 483-497. MR.1242434 (94j:54017)

[48] M. van de Vel, Collapsible polyhedra and median spaces, Proc. Amer. Math. Soc. 126 (1998), no. 9, 2811-2818. MR 1452832 (98k:57041)

[49] E. R. Verheul, Multimedians in Metric and Normed Spaces, CWI Tract, vol. 91, Stichting Mathematisch Centrum, Centrum voor Wiskunde en Informatica, Amsterdam, 1993. MR.1244813(94i:54062)

[50] J. H. C. Whitehead, Note on the condition n-colc, Michigan Math. J. 4 (1957), 25-26. MR.0087092(19:300h)

[51] R. E. Williamson, Cobordism of combinatorial manifolds, Ann. Math. 83 (1966), 1-33. MR0184242(32:1715) 
[52] N. Witte, Entfaltung simplizialer Sphären, Technischen Universität Berlin, 2004. Diplomarbeit (Master Thesis).

[53] E. C. Zeeman, On the dunce hat, Topology 2 (1964), 341-358. MR0156351 (27:6275)

[54] Li Zhongmou, Every 3-manifold with boundary embeds in Triod $\times$ Triod $\times I$, Proc. Amer. Math. Soc. 122 (1994), no. 2, 575-579. MR1254861(95b:57015)

Steklov Mathematical Institute of the Russian Academy of Sciences, ul. Gubkina 8, Moscow 119991, Russia

E-mail address: melikhov@mi.ras.ru

FACUlty of Mathematics, Informatics, And Mechanics, University of Warsaw, BANACHa 2, 02-097 WarszaWA, Poland

E-mail address: jzajac@mimuw.edu.pl 